\title{
Building the BEaTriX facility for the ATHENA mirror modules X-ray testing
}

Salmaso, B.; Basso, S.; Cotroneo, V.; Ghigo, M.; Pareschi, G.; Redaelli, E.; Sironi, G.; Spiga, D.; Tagliaferri, G.; Vecchi, G.

Total number of authors:

25

Published in:

Proceedings of SPIE

Link to article, DOI:

$10.1117 / 12.2594182$

Publication date:

2021

Document Version

Publisher's PDF, also known as Version of record

Link back to DTU Orbit

Citation (APA):

Salmaso, B., Basso, S., Cotroneo, V., Ghigo, M., Pareschi, G., Redaelli, E., Sironi, G., Spiga, D., Tagliaferri, G., Vecchi, G., Fiorini, M., Incorvaia, S., Úslenghi, M., Paoletti, L., Ferrari, C., Zappettini, A., Lolli, R., Sanchez Del Rio, M., Burwitz, V., ... Ferreira, I. (2021). Building the BEaTriX facility for the ATHENA mirror modules X-ray testing. In S. L. O'Dell, J. A. Gaskin, \& G. Pareschi (Eds.), Proceedings of SPIE: Optics for EUV, X-Ray, and Gamma-Ray Astronomy X [118220M] SPIE - International Society for Optical Engineering. Proceedings of SPIE - The International Society for Optical Engineering Vol. 11822 https://doi.org/10.1117/12.2594182

\section{General rights}

Copyright and moral rights for the publications made accessible in the public portal are retained by the authors and/or other copyright owners and it is a condition of accessing publications that users recognise and abide by the legal requirements associated with these rights.

- Users may download and print one copy of any publication from the public portal for the purpose of private study or research.

- You may not further distribute the material or use it for any profit-making activity or commercial gain

- You may freely distribute the URL identifying the publication in the public portal 


\title{
Building the BEaTriX facility for the ATHENA mirror modules X-ray testing
}

\author{
B. Salmaso ${ }^{\text {1a }}$, S. Basso ${ }^{\text {a }}$, V. Cotroneo ${ }^{\text {a }}$, M. Ghigo ${ }^{\text {a }}$, G. Pareschi ${ }^{\text {a }}$, E. Redaelli ${ }^{\text {a }}$, G. Sironi ${ }^{\text {a }}$, D. Spiga ${ }^{\text {a }}$, \\ G. Tagliaferri ${ }^{\mathrm{a}}$, G. Vecchi ${ }^{\mathrm{a}}$, M. Fiorini ${ }^{\mathrm{b}}$, S. Incorvaia ${ }^{\mathrm{b}}$, M. Uslenghi ${ }^{\mathrm{b}}$, L. Paoletti ${ }^{\mathrm{c}}$, C. Ferrari ${ }^{\mathrm{d}, \mathrm{a}}$, A. \\ Zappettini $^{\text {d }}$, R. Lolli ${ }^{\mathrm{e}}$, M. Sanchez del Rio ${ }^{\mathrm{f}}$, V. Burwitz ${ }^{\mathrm{g}}$, F. Christensen $^{\mathrm{h}}$, D. D. Ferreira ${ }^{\mathrm{h}}$, N. C. \\ Gellert $^{\text {h }}$, S. Massahi ${ }^{\text {h }}$, M. Bavdaz ${ }^{\text {i }}$ I. Ferreira ${ }^{\text {i }}$ \\ ${ }^{a}$ INAF Astronomical Observatory Brera, Via E. Bianchi 46, 23807 Merate, Lecco (Italy) \\ ${ }^{\mathrm{b}}$ INAF-IASF Milano, Via A. Corti 12, 40133 Milano (Italy) \\ ${ }^{\mathrm{C}}$ INAF Astronomical Observatory Padova, Vicolo Osservatorio 5, 35122 Padova (Italy) \\ ${ }^{\mathrm{d}}$ CNR-IMEM, Parco Area delle Scienze 37/A, 43124 Parma (Italy) \\ ${ }^{e}$ Department of Physics and Earth Science, Ferrara University, Via Saragat 1, 44122 Ferrara (Italy) \\ ${ }^{\mathrm{f}}$ European Synchrotron Radiation Facility, B.P. 220, 38043 Grenoble (France) \\ ${ }^{\mathrm{g}}$ Max-Planck-Institüt für extraterrestrische Physik, Garching (Germany) \\ ${ }^{\mathrm{h}}$ DTU-space, Juliane Maries Vej 30, DK-2100 Copenhagen (Denmark) \\ ${ }^{\mathrm{i}}$ ESTEC, European Space Agency, Keplerlaan 1, 2201 AZ Noordwijk (Netherlands)
}

\begin{abstract}
BEaTriX (Beam Expander Testing X-ray) is the X-ray facility under construction at the INAF-Osservatorio Astronomico Brera (Merate, Italy) to prove that it is possible to perform the X-ray acceptance tests (PSF and Aeff) of the ATHENA mirror modules at the required rate and with the required accuracy. The unique optical setup makes use of a micro-focus X-ray source with anode in Titanium, a paraboloidal mirror with small radius of curvature, and a set of crystals to monochromate and expand the beam to fully illuminate the entrance pupil of the ATHENA MMs. The quality of the optical components, and their precise alignment, guarantees the production of a parallel beam at $4.51 \mathrm{keV}$, to be extended in a second phase to $1.49 \mathrm{keV}$ able to perform the acceptance requirements for the ATHENA MMs. The facility is completed and the alignment of the various components is now ongoing and expected to be completed in fall this year, with the X-ray beam fully qualified. Then the commissioning will start with the tests on the ATHENA MMs. In this paper, we present the current status.
\end{abstract}

Keywords: ATHENA, BEaTriX, X-ray testing, X-ray microfocus source, beam expander, asymmetric diffraction, crystals

\section{INTRODUCTION}

ATHENA (Advanced Telescope for High-ENergy Astrophysics) is a Large X-ray mission selected by ESA within the Cosmic Vision Program [1], with launch foreseen in early 2030's. The observatory will study the formation of galaxy clusters and the growth of black holes. These science goals drive very demanding requirements. The entire optics should have a focal length of $12 \mathrm{~m}$, a Point Spread Function (PSF) with Half Energy Width (HEW) of 5 arcsec at the energy of $1 \mathrm{keV}$, and an effective area of about $1.4 \mathrm{~m}^{2}$ [2].

A very large aperture X-ray mirror needs to be built, with diameter of about $2.5 \mathrm{~m}$ [3], using the novel Silicon Pore Optics (SPO) technology, developed at Cosine (https://www.cosine.nl/, The Netherlands). Due to the diameter, a modular approach is foreseen, where each step of the process has to be followed by dedicated tests and calibration procedures. In a typical SPO manufacturing process, 38 silicon plates with rectangular grooves are stacked, with dedicated robotic machines. Two stacks are aligned to form an X-ray Optical Unit (XOU), working in double reflection

\footnotetext{
${ }^{1}$ bianca.salmaso@,inaf.it; phone +39-02-72320428; www.brera.inaf.it
} 
and so reconstructing the Wolter-I focusing geometry. Two XOUs are aligned and integrated in parallel to form an SPO Mirror Module (MM). The alignment is performed using synchrotron radiation at the XPBF 2.0 beamline at the BESSY II facility in Berlin, Germany [4]. The MMs are, at present, characterized at XPBF 2.0 and at the $123 \mathrm{~m}$-long PANTER facility of MPE near Munich [5]. Neither of the two can be used to routinely test $600 \mathrm{MMs}$ for acceptance and integration into the Mirror Assembly Module (MAM).

Therefore, INAF-OAB has proposed to the community a new facility, with two beam lines working at the energies of $1.49 \mathrm{keV}$ and $4.51 \mathrm{keV}$ [6-7]. The first beamline under realization is the $4.51 \mathrm{keV}$. The system is designed as a compact screening facility with fast vacuum pump-down (differently from large facility such as the PANTER), and a broad beam to fully illuminate the aperture of the MM under test; this makes BEaTriX different from other light sources, such as synchrotrons, where the X-ray beam is very narrow and needs to be scanned throughout the MM aperture.

The design and construction of this facility is supported and funded by ESA, by the AHEAD consortium, by ASI and by INAF. A team of scientists is working together to realize this facility, with the Italian leadership by INAF (OABrera + OAPd + IASF-Mi); the team includes CNR-IMEM (Istituto dei Materiali per l'Elettronica e il Magnetismo), ESRF (European Synchrotron Radiation Facility in Grenoble), MPE (Max Planck Institute for Extraterrestrial Physics in Garching), DTU (Technical University of Denmark) and ESA.

The optical and mechanical design of BEaTriX (Fig. 1) was already presented [6]: it encompasses an X-ray microfocus source with a Titanium anode (A), placed in the focus of a paraboloidal mirror (C), a monochromation stage with symmetrically-cut crystals (D), and an expansion stage (E) where the beam is expanded by an asymmetrically-cut crystal to a $170 \times 60 \mathrm{~mm}$ size. The beam is so collected by the MMs under test, present in the chamber $(\mathrm{F})$ and focused on a CCD camera placed at a $12 \mathrm{~m}$ distance $(\mathrm{H})$. The optical components are moved and aligned by means of precise vacuum motors. All the system is enclosed in a vacuum tank reaching $10^{-6} \mathrm{mbar}$, divided into several compartments, separated by gate valves. Each compartment can be evacuated and vented separately in order to minimize the time required for the MM mounting and removal.
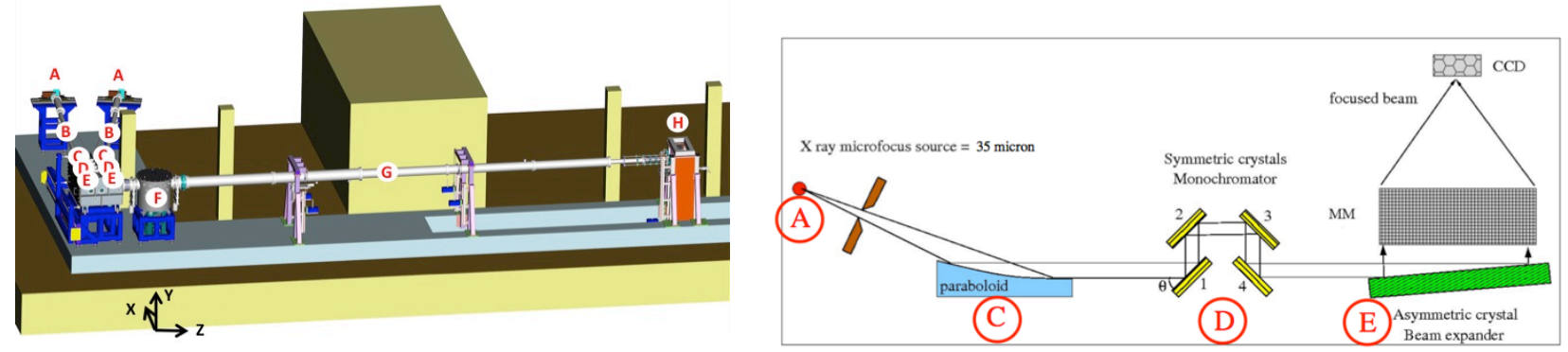

Figure 1: BEaTriX: mechanical design (left), and optical design (right)

The realization of the mechanical and optical components, for the first beam line at $4.51 \mathrm{keV}$, is supported by a number of companies, working in close contact with INAF-OAB. The vacuum system, whose design was already presented in a previous paper [6], was realized and installed in Merate in summer/autumn 2020. The divergent beam emerging from the source is made parallel and expanded by the following optical components: a paraboloidal mirror entirely polished at INAF-OAB, coated at DTU and calibrated at PANTER [8]; a monochromation stage is composed by 4 symmetrically cut crystals, tested by IMEM-CNR (https://www.imem.cnr.it/); an expansion stage where the beam is diffracted and expanded by an asymmetrically-cut crystal, realized and tested at IMEM-CNR. The optical components are aligned with the help of vacuum motors compatible up to $10^{-6} \mathrm{mbar}$, and with the assistance of a wave front sensor in collaboration with LOA and Imagine Optic (https://www.imagine-optic.com/). At $12 \mathrm{~m}$ distance from the MMs under test, a directly-illuminated CCD camera is present. The camera can move on three linear stages actuated by servomotor, equipped with absolute encoders. The MMs can be tested in the range $20 \pm 25{ }^{\circ} \mathrm{C}$ by means of a thermal box surrounding the MM itself in order to measure the PSF as function of the MM temperature. The thermal box was entirely realized by $\mathrm{OAB}$, and it is connected to a dedicated chilling system. The driving software was entirely realized by INAF-OAB, using the LabVIEW software.

This paper presents the advancement status in the realization of the BEaTriX facility. All the optical components are now available in Merate premises, and the alignment of the optical components is on going. The beam qualification is expected to be completed in fall this year. Then the commissioning will start with the tests on the ATHENA MMs. 


\section{THE VACUUM SYSTEM}

Following the design realized by Tecnomotive (Fig. 1 left) [6], the vacuum system was produced, integrated and installed in Merate by Kenosistec (https://www.kenosistec.com/en/). The installation and the mechanical alignment of the parts required the realization of tools ad hoc developed by Kenosistec. The system is installed on an L-shaped massive block of concrete [Fig. 2] on top of damping polymer pads in order to isolate the system from ground vibrations [6].

The vacuum pumping system was also designed by Tecnomotive, and installed by Kenosistec. The driving software was entirely realized by INAF-OAB (Fig. 3). The vacuum system is divided into several compartments, separated by gate valves procured by Allectra (https://www.allectra.com/).

The vacuum is reached by a system of scroll pumps and turbo-molecular pumps procured by Leybold (https://www.leybold.com/it/it/). All the pumps are oil free to avoid contamination. A Scrollvac 18plus is dedicated to the fore vacuum; an Ecodry 40plus is installed for the high vacuum line; turbo pumps, magnetically levitated to avoid vibrations (TMP $300 \mathrm{l} / \mathrm{s}$ and TMP $600 \mathrm{l} / \mathrm{s}$ ) allow to reach $10^{-6} \mathrm{mbar}$. The vacuum is controlled by electro-valves and measured by sensors procured by Pfeiffer (https://www.pfeiffer-vacuum.com/en/). The electrical cabling of the facility was realized by INAF-IASF.

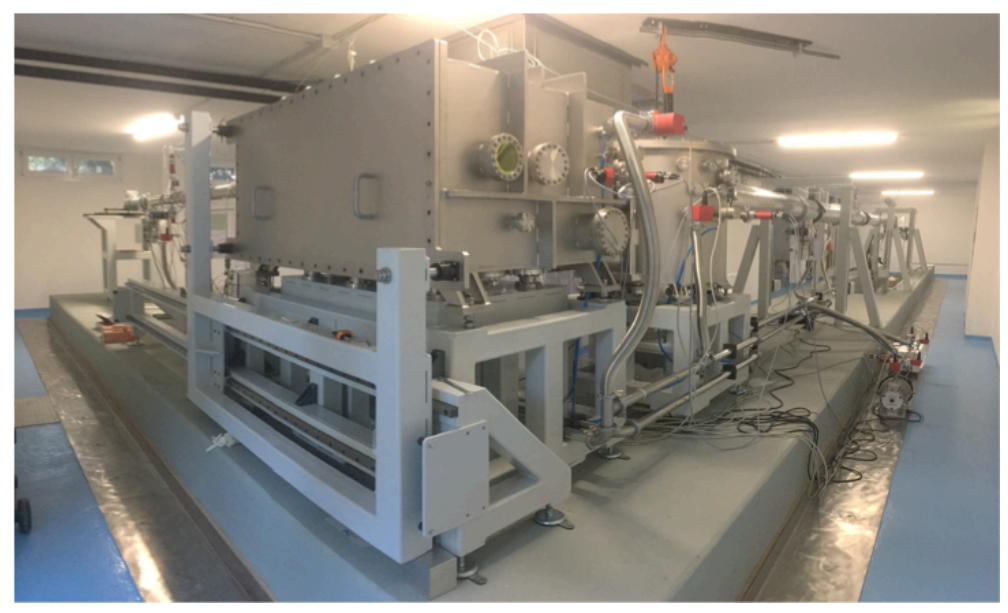

Figure 2: The BEaTriX vacuum system

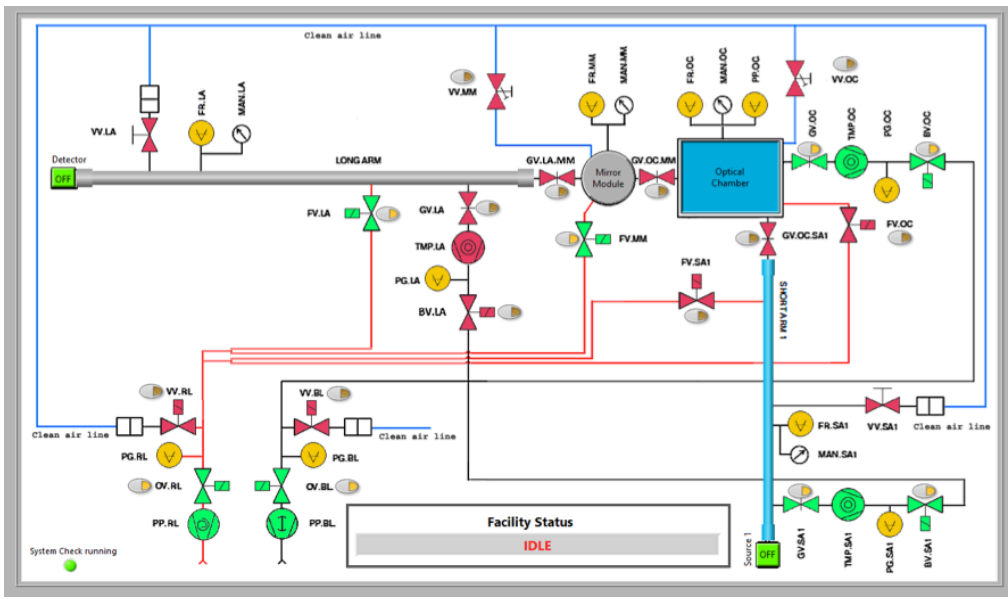

Figure 3: The vacuum pumping and venting layout 
After installation, a leak test was performed by Kenosistec to ensure the vacuum seal. A software utility, "Rate of Pressure Raise", has been developed in LabVIEW by OAB, and was used together with the helium leak detector tests, in order to give a reference pressure raise curve to be used during the life of BEaTriX.

The venting is performed with clean dehumidified air of Class 1.2.1 according to ISO 8573.

Preliminary measurements, on the MM chamber, were performed to measure the evacuation time needed to go from 1000 to $10^{-4}$ mbar: the result was $15 \mathrm{~min}$, with empty chamber. The result fulfills with margin, the requirement for the throughput of $3 \mathrm{MM} /$ day (evacuation in 30min). The venting to atmospheric pressure was reached in less than 6 min. To assure a fast operation of the facility, the MM chamber is also equipped with a door with a fast closing mechanism, which ensures in turn a safe release of the vacuum when venting at overpressure.

\section{THE DETECTOR TOWER}

The detector tower hosts the detector and its motorizations. The detector is connected to the vacuum system by a bellow to enable the focus search (Fig. 4 right). During the installation, the tower was positioned at three focal distances to drill holes (Fig. 4 left) for the tower anchoring at $\mathrm{f}=12-10-8 \mathrm{~m}$, for future flexibility. The tower is now fixed to the $\mathrm{f}=12 \mathrm{~m}$ position.
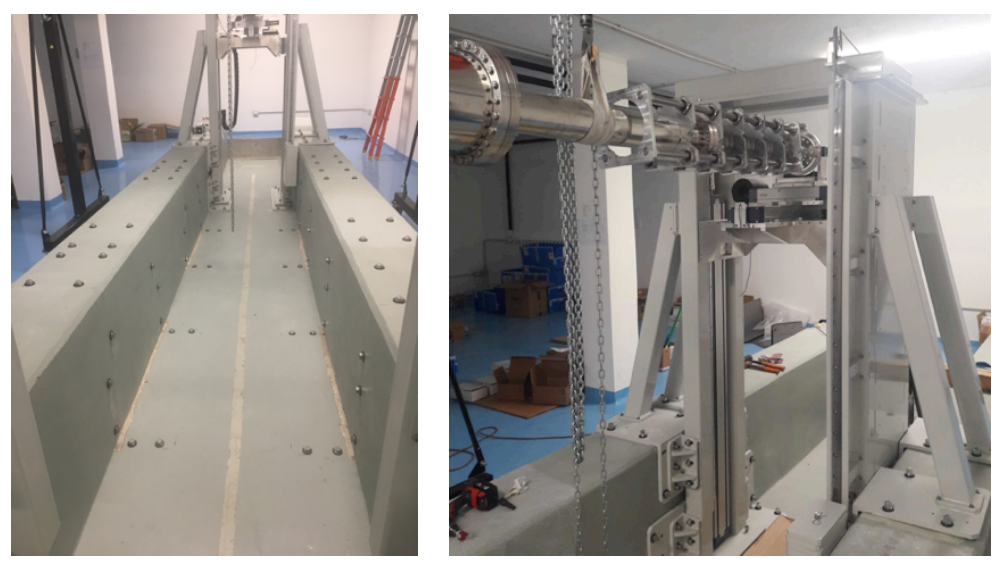

Figure 4: The detector tower. Left: holes to anchor the tower are prepared at $f=12-10-8 \mathrm{~m}$, for flexibility of the facility. Right: The tower with the bellow for the focus search.

The detector moves on three linear stages produced by Rollon and motorized by Omron. The travel ranges of the stages are: range $\mathrm{Z}=600 \mathrm{~mm}$ (for the focus search), range- $\mathrm{Y}=1500 \mathrm{~mm}$ (for the vertical displacement of the image for MMs of different radii), range- $X=200 \mathrm{~mm}$ (for the qualification of the beam).

The movement of the detector is controlled by a LabVIEW routine, developed by INAF-OAB. For hardware reasons, the $\mathrm{Y}$ and $\mathrm{Z}$ limits are dependent on each other: the LabVIEW software tracks the position of the CCD (the red dot in Fig. 5) within the permitted region. A camera is monitoring the CCD position directly from the control desk.

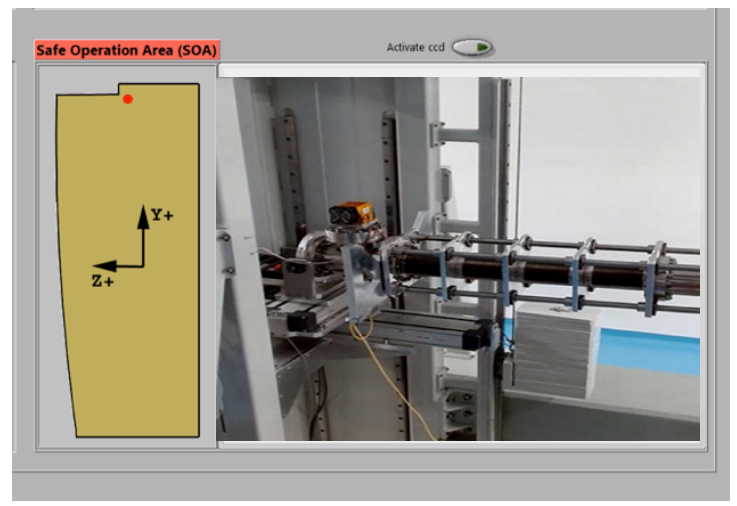

Figure 5: The permitted area for YZ movement of the CCD, and the image taken by the camera monitoring the CCD position 


\section{THE X-RAY SOURCE}

The X-ray source in use is a compact micro-focus source with titanium anode, manufactured by Incoatec (Germany). The source is air-cooled and injects X-rays directly in vacuum. The X-ray spot was measured, at Incoatec site, to have a gaussian profile with a $35 \mu \mathrm{m}$ FWHM. The beam flux in the titanium $\mathrm{K}_{\alpha}$ doublet $(4.511 \mathrm{keV})$ is copious, on the order of $10^{11}$ photons/s/sterad for a dissipated power of $1 \mathrm{~W}$. The source is enclosed in a shielding box, manufactured by Gilardoni (Italy) that avoid X-ray leaks in the operation environment. The driving software was written in LabVIEW by INAF-OAB with the support of Incoatec.
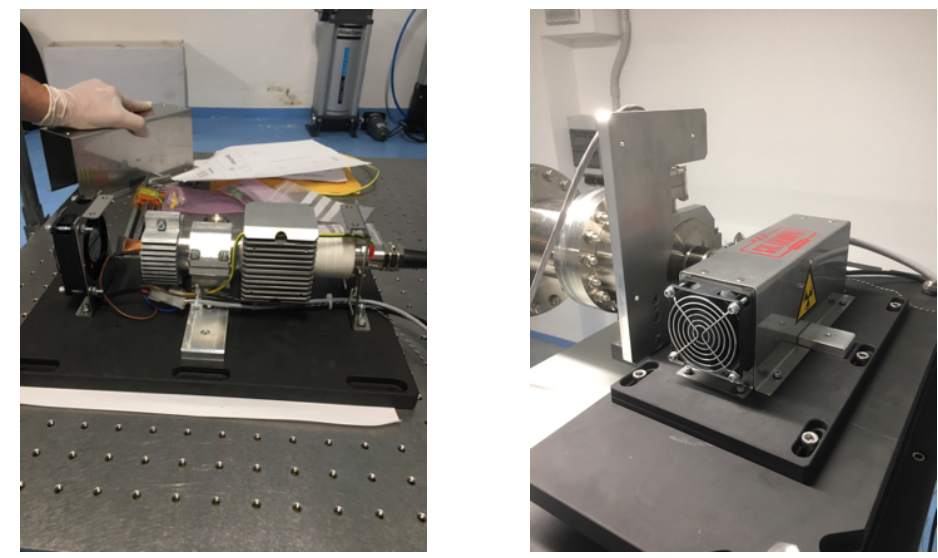

Figure 6: The X-ray tube with fan and radiators (left), mounted with the X-ray shield on its manipulator (right).

The source is placed on a manipulator that assists the alignment of the source itself with respect to the paraboloidal mirror. A pinhole is placed in front of the micro-focus X-ray source. It consists of a small $(1 / 2 \mathrm{inch})$ tungsten disk, produced by Amptek, with a $2.2 \mathrm{~mm}$ thickness and a $400 \mu \mathrm{m}$ diameter. It limits the beam along the short arm of the facility, avoiding the X-ray incidence on the tube walls, which might cause unwanted X-ray reflection/scattering or diffuse background. At the same time, the pinhole located near the X-ray source provides visual reference for the parabolic mirror alignment. The scattered/reflected radiation off the pinhole, measured in the BEaTriX facility, was hardly detectable (scattering FWHM $<0.02$ deg, reflectivity $<0.0001$ ), proving that the pinhole is suitable for the collimation of the X-ray beam in the short arm of BEaTriX.

Just after the pinhole, there is an X-ray shutter (Uniblitz, XRS25). It is connected, via a coaxial cable, to the CCD camera placed at the other end of the system, which gives the synchronization signal to the shutter in order to close it during the frame readout.

A Si-PIN detector (Amptek, X123 with $25 \mathrm{~mm}^{2}$ area / $500 \mu \mathrm{m}$ thickness / $25 \mu \mathrm{m}$ Be window), placed in front of the paraboloidal mirror, monitors the flux stability of the source (Fig. 17C).

\section{THE PARABOLOIDAL MIRROR}

The X-ray source is placed in the focus of a paraboloidal mirror. The surface quality of the mirror is essential to create a parallel X-ray beam at the entrance of the monochromation stage, which in turns is crucial to minimize the beam expander dispersion in wavelength [10]. The fused quartz mirror substrate was procured by Zeiss in a preliminary lapped state. The polishing and figuring was then performed at INAF-OAB in several iterations [8]. The Zeeko IRP 1200 machine, operated at $\mathrm{OAB}$, has been used to remove the surface damage from the mirror as procured, and to reduce the figure and roughness within specific tolerances: both a bonnet and a pitch-polishing tool have been used. Our IBF machine has been subsequently used to correct the low-frequency errors introduced by the pitch process. The performance of each polishing/figuring run has been monitored using the MPR (for shape) and CCI (for roughness) metrology tools available at Media Lario (Bosisio Parini, Italy) coupled with instrument available in OAB, CMM for radius of curvature determination and MFT for roughness caracterization. The reflective coating was deposited at the Denmark Technical University (DTU, Copenhagen, Denmark) to enhance the reflectivity at $4.5 \mathrm{keV}$ using a bi-layer of $\mathrm{Cr}(4.6 \mathrm{~nm})$ and Pt $(30 \mathrm{~nm})$. X-ray tests were performed at the PANTER X-ray facility (Max Plank Institute for 
Extraterrestrial Physics, MPE Garching, Germany), before and after the coating, using both the TRoPIC and PIXI cameras. The tests have been performed at $1.49 \mathrm{keV}$ and $4.51 \mathrm{keV}$ X-ray energies, using the classical "diverging beam" setup. At $1.49 \mathrm{keV}$ it was possible to work also in the parallel beam configuration, by means of a Fresnel zone-plate available at PANTER [9]. The PSF at $1.49 \mathrm{keV}$ gave an HEW value of about 3 arcsec, both before and after coating (Fig. 6B and 6C), as measured with the parallel beam setup and the PIXI detector (20 $\mu \mathrm{m}$ pixel, corresponding to $\sim 0.87$ arcsec). At $4.51 \mathrm{keV}$, only the measurements with the divergent beam configuration and the TRoPIC detector $(75 \mu \mathrm{m}$ pixel, corresponding to $\sim 3.3 \mathrm{arcsec}$ ) were possible, giving a result of $\mathrm{HEW}_{\text {div-4.5-TRoPIC }}=6.5 \mathrm{arcsec}$, to be compared with the $\mathrm{HEW}_{\text {div-1.5-TRoPIC }}=5.1 \mathrm{arcsec}$ at the lower energy. The $1.5 \operatorname{arcsec}$ increase in the HEW, going from 1.49 to 4.51 $\mathrm{keV}$, is attributed to lower quality areas, of the mirror surface, illuminated in the PANTER campaign but not used in the BEaTriX beam moderation: from the PANTER campaign, an upper limit of $1.5 \operatorname{arcsec}$ contribution of scattering at 4.51 $\mathrm{keV}$ could be fixed; anyway, we cannot exclude that the HEW remains unchanged, going from 1.49 to $4.51 \mathrm{keV}$, for what concerns the BEaTriX setup [8].

The focal length was measured in the range $4958 \div 4952 \mathrm{~mm}$, to be compared with the nominal focal length of 4959 $\mathrm{mm}$, assumed from the center of the mirror. From this value, the mechanical positioning of the mirror was defined.

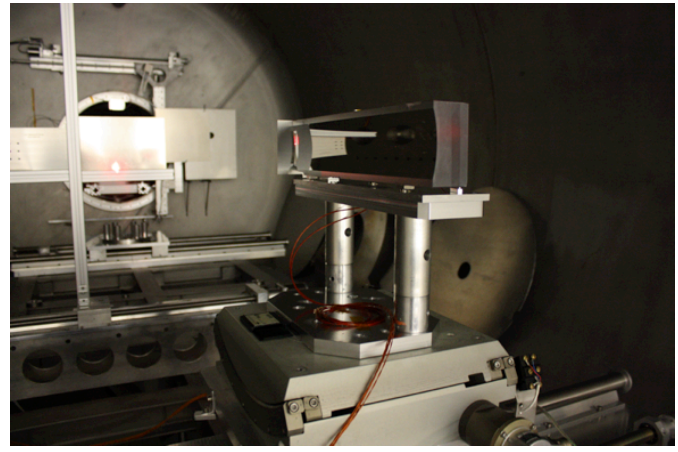

(A)

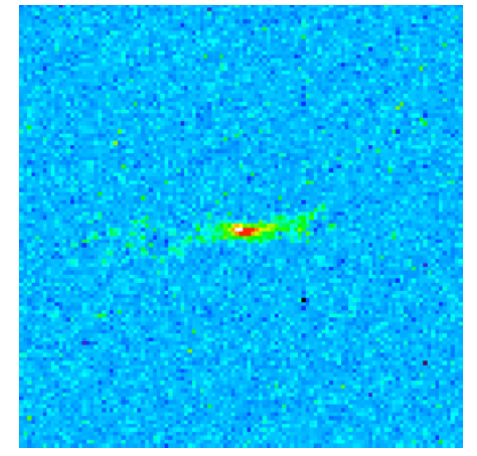

(B)

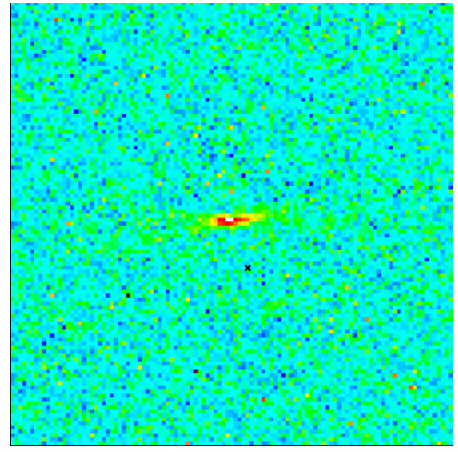

(C)

Figure 7: A) the BEaTriX paraboloidal mirror mounted in the PANTER vacuum chamber, viewed from the detectors side. B) Best focus measured with PIXI at $1.49 \mathrm{keV}$ in parallel setup, before coating, HEW = 3.1 arcsec. C) Same as B) but after coating, HEW= 2.8 arcsec.

\section{THE CRYSTALS}

After the collimation, the beam is filtered tightly in energy to ensure the final collimation of the expanded beam. This is obtained via a 4-fold diffraction on silicon crystals, cut parallel to the (220) planes. At each diffraction, only photons with the energy fulfilling the Bragg condition are reflected: in this way, the $\mathrm{K}_{\alpha 1}$ fluorescence line at $4.51 \mathrm{keV}$ can be isolated from the source spectrum; iterating the diffraction 4 times makes the passing beam more monochromatic. The crystals are organized into 2 pairs, each of them cut out from a single silicon block aiming at the alignment of their crystalline planes, a geometry known as channel-cut crystal (CCC). The two CCCs can be rotated to de-tune the Bragg energy slightly and so improve the monochromation to the required values [6]. The CCCs were realized by Crystal Scientific (Whittingham, UK) and tested by IMEM-CNR (Parma, Italy). Measurements with an X-ray diffractometer at $8.045 \mathrm{keV}$, properly filtered by a 4-bounce Bartel monochromator, were performed in both single and double diffraction. The results revealed the presence of some mosaicity at the border of the diffractive surfaces (Fig. 8B), probably due to a residual surface damage left after cutting the channel. A re-etching was therefore performed. The comparison of the diffraction profiles, before and after etching, is shown in Fig. 8B-8C, for the $\mathrm{X}$ direction; similar improvement was measured in the $\mathrm{Y}$ direction. The measurements demonstrate that the mosaicity has been removed as the scattering tails have been damped out.

The beam expander is based on a silicon crystal, cut asymmetrically, i.e., at approximately 45 deg with respect to the (220) crystalline planes. This concept was proposed by Christensen et al. in 1994 [11]; however, the beam expansion was limited to a single direction, and the divergence obtained was much larger than what aimed with BEaTriX. Monochromatic X-rays, incident at a grazing angle (but larger than the total reflection one), are spread over the $170 \mathrm{~mm}$ $\times 60 \mathrm{~mm}$ surface of the silicon crystal and are so diffracted at a nearly-right angle. In this way, we obtain a parallel 
beam as wide as the asymmetric crystal, covering the entrance pupil of the SPO MMs for ATHENA. Fig. 9 shows the beam expander crystal: its characterization is already reported in [6].

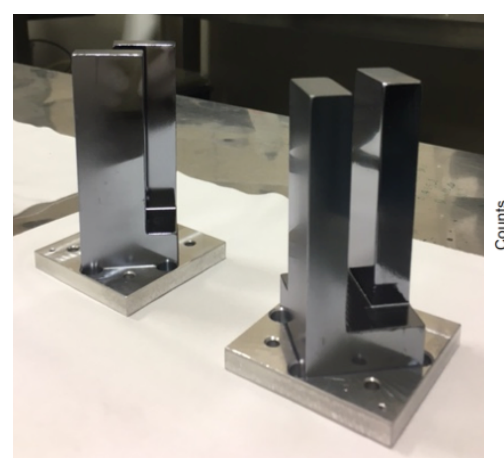

(A)

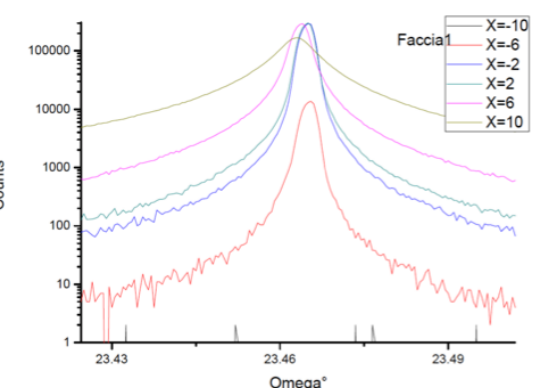

(B)

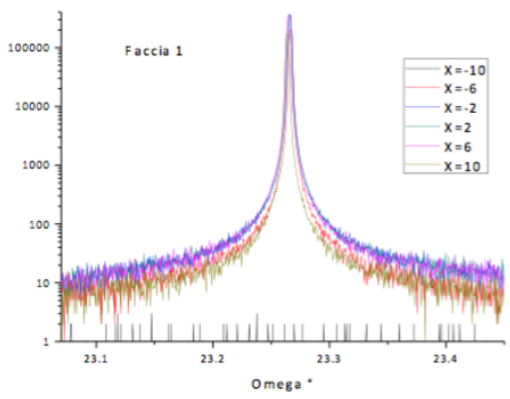

(C)

Figure 8: A) The CCCs glued on their supports. Rocking curve before (B) and after (C) the etching, in the X direction (corresponding to the direction of the propagating beam)

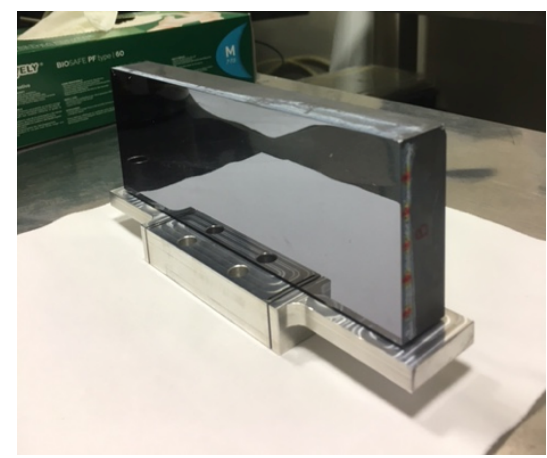

Figure 9: The Beam Expander crystal, glued on it support.

\section{THE VACUUM MOTORS}

The optical components are moved and aligned by means of precise vacuum motors produced by Physik Instrumente (Germany). The stages were integrated by INAF-OAB to equip the optical components with the degree of freedom necessary for the beam alignment. Several PT100 sensors are installed in the vacuum chambers to monitor the temperature of the optical components and their motorizations.

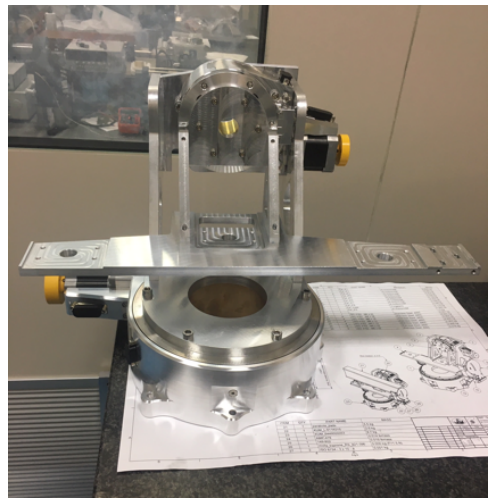

(A)

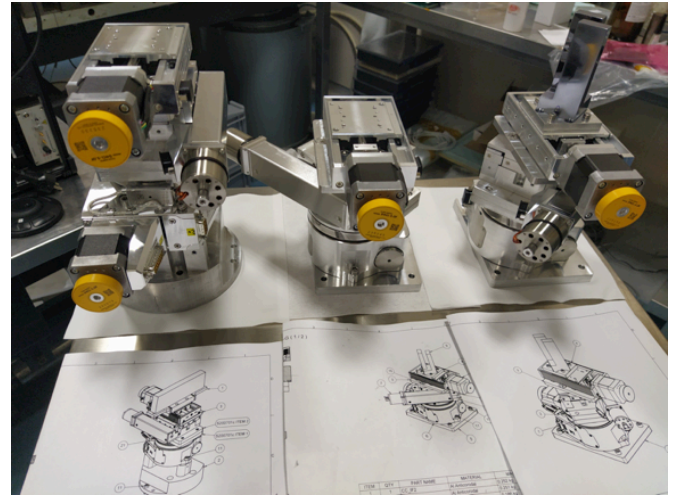

(B)

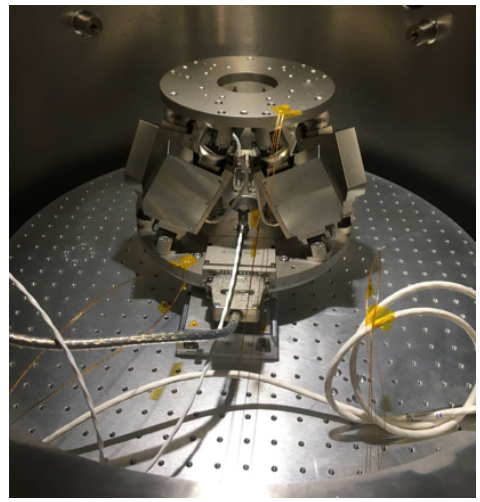

(C)

Figure 10: The assembled motorization stages for the parabolic mirror (A), CCCs and Beam Expander $(B), M M(C)$

The MM is motorized in vacuum with the hexapod H-824.G2V (C), which is mounted on a translational stage, used to bring a reference area into the beam for the Effective Area measurements. 
The temperature reached in vacuum, for the hexapod, was measured keeping the motor powered for 24h. Two temperature sensors (PT100) have been installed at the top and base of the hexapod. The sensor installed at the base is closed to the connector containing a board. One other sensor was positioned in the MM Chamber, measuring the air temperature: in the final configuration, this sensor will measure the temperature close to the MM. A max temperature overtime increase of $4^{\circ} \mathrm{C}$, from 21 to $25^{\circ} \mathrm{C}$, was measured at the bottom plate. The very low increase, the distance of the MM from the hexapod base plate and the insulating support in titanium ensure that no heat to the MM can occur due to the hexapod operation.

\section{THE MIRROR MODULE INTERFACE}

The interfacing structure that supports the MM is made of titanium to ensure a low conductive link from the hexapod and to reduce the CTE mismatch. The upper part of the structure, holding the MM, can be adjusted to fit all the different modules. In order to avoid any relevant deformation and problems related to possible printed scratches on the Invar brackets of the module, a system design based on a magnetic coupling has been developed. Six magnets made in Neodymium will keep the module in the correct position. Safety mechanical stoppers can be easily added-on.

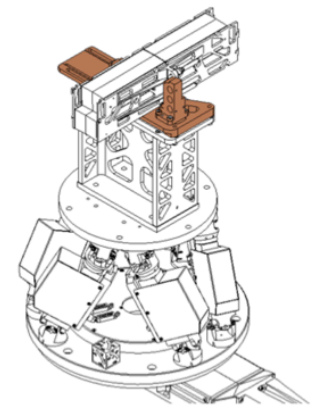

(A)

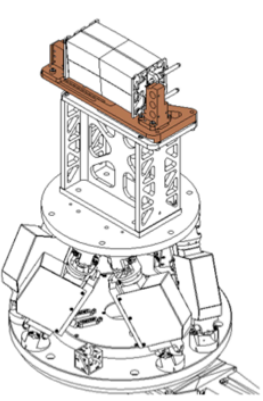

(B)

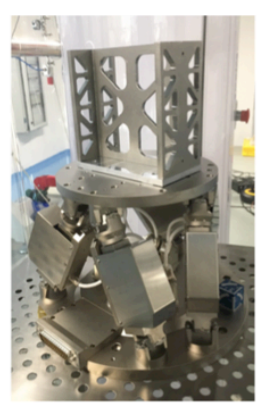

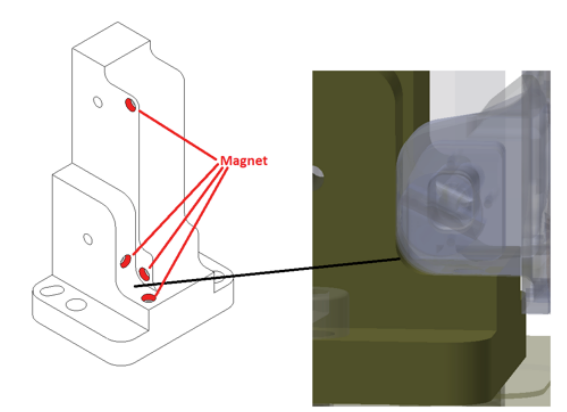

(C)

Figure 11: A) The interface with all the modules is composed by 3 titanium parts. One of them can be adjusted to fit all the different modules. B) Status of the realization. $C$ ) detail of the magnetic clamping

\section{THE THERMAL BOX}

A thermal box was designed [12] and manufactured by INAF-OAB, to surround the MMs and permit the measurement of their PSF in the range $20 \pm 25^{\circ} \mathrm{C}$. The thermal box is connected to the RP245E chilling system, produced by the Lauda firm (https://www.lauda.de/en/). The box is realized in Aluminum. The surfaces were anodized with ALUMITE, a layer with high emissivity in the infrared region, with typical values of emissivity greater than 0.9 in the $2-30 \mu \mathrm{m}$ range and greater than 0.85 in the spectral range below $10 \mu \mathrm{m}$. Due to difficulties in the protection procedure, the anodization was performed on all the surfaces and was removed by milling on the surfaces requiring a low thermal emission. Tests were performed to certify the selected chiller and liquid, assessing their capability to reach the needed temperatures.
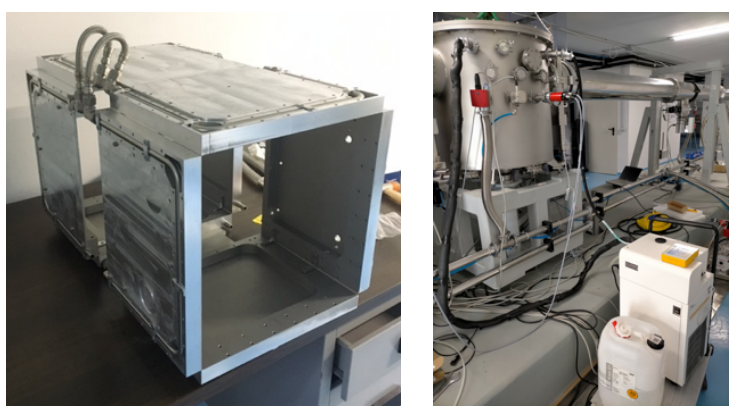

Figure 12. Left: The thermal box. Right: the rear of the MM chamber with the chiller connected to the feed-throughts. 


\section{THE CCD AND THE COLD FINGER}

The camera is an iKon-L from Andor, Model DW936R, mounting a 2048x2048 pixels (13.5 um x 13.5 um pixel size) back-illuminated sensor from E2V, model CCD42-40, in open configuration. Before installation, the camera was tested at INAF-IASF: the sensor was maintained at room temperature, and in nitrogen atmosphere. Both measurements with dark current and illuminating the sensor with visible light were successfully performed.

Before installing the camera in BEaTriX, and cooling the sensor to the operating temperature of $-20^{\circ} \mathrm{C}$, a "cold finger" was realized and tested at INAF-IASF. The cold finger was installed at the end of the long arm where the camera will be flanged, to avoid any potential condensation on the CCD sensor. During the test in vacuum, it reached $-30^{\circ} \mathrm{C}$ in $1 \mathrm{~h}$.

The CCD was then installed in the BEaTriX vacuum system for the incoming tests: the linearity of the dark-current signals versus exposure time and temperature was measured, and the photon transfer curve was measured using the dark current signal to estimate gain and readout noise at $-20^{\circ} \mathrm{C}$. All the measurements resulted in accordance with specification.

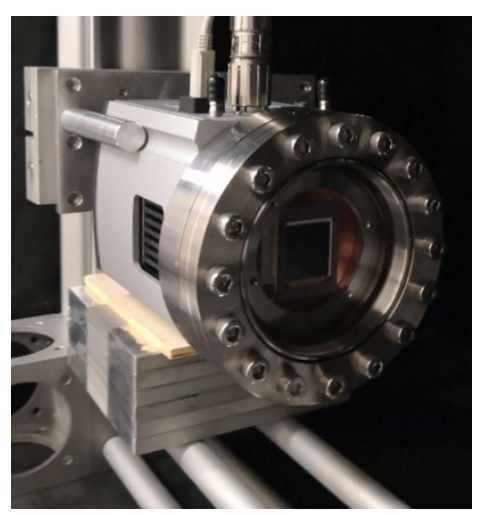

(A)

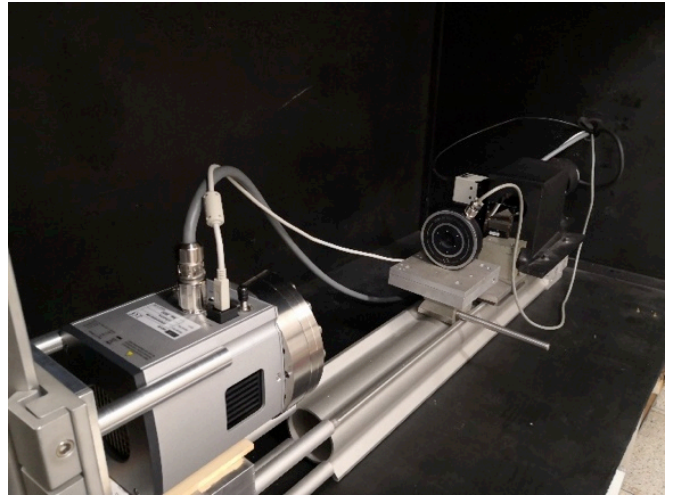

(B)

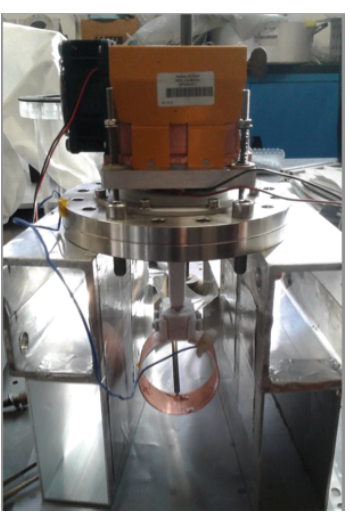

(C)

Figure 13. A) the camera ready for the tests at room temperature. B) setup for measurements with visible light. C) the cold finger before the installation in the vacuum system.

\section{THE CLEAN TENT}

In order to handle the MMs in a clean environment during the loading/unloading into the facility, the front door of the MM chamber opens into an ISO5 clean tent. The tent was realized and installed by Bema Technology srl (Sovico, MB, Italy), and the ISO5 qualification tests were successfully performed by CAMST-EMS.
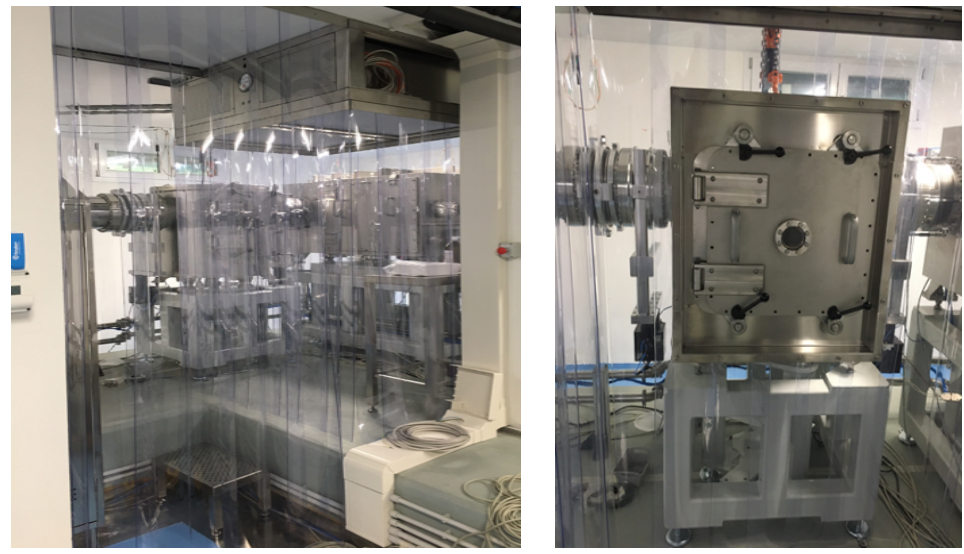

Figure 14. Left) the ISO5 clean tent. Right: the MM door opens into the clean environment 


\section{THE SOFTWARE}

The Control software was developed by INAF-OAB in LabVIEW. The structure of the software foresees two independent sections: the "Main" section and the "Maintenance" section. The Main section is the place where all the normal activities for the control and usage of the facility are located. The Maintenance section is the place where is it possible to manually control every component of the facility. An example of the Maintenance section is shown in Fig. 15 , presenting the "Valves and vacuum sensors" page and the facility status during the pumping down: the blue color of the Short Arm and Optical Chamber indicates the reaching of $10^{-4}$ mbar. Control pages are developed for all the following components: pumps, valves and vacuum sensors, X-ray source, detector, motors (in vacuum and in air), flux monitor, X-ray source. Two routines are developed for special controls on the facility: the "Rate of Raise" is developed to control possible leakage in the vacuum system; the "Optical Chamber alignment and testing" is used to control the vacuum of the Optical Chamber and Short Arm, during the alignment of the optical components.

All the BEaTriX facility is under a UPS battery of $20 \mathrm{~kW}$, which will start to work in case of power failure. To avoid an uncontrolled shut down of the system when the battery power will end, the computer is connected to the UPS battery and starts the facility shut down with a delay of $8 \mathrm{~min}$ from the start of the operation of the UPS battery.

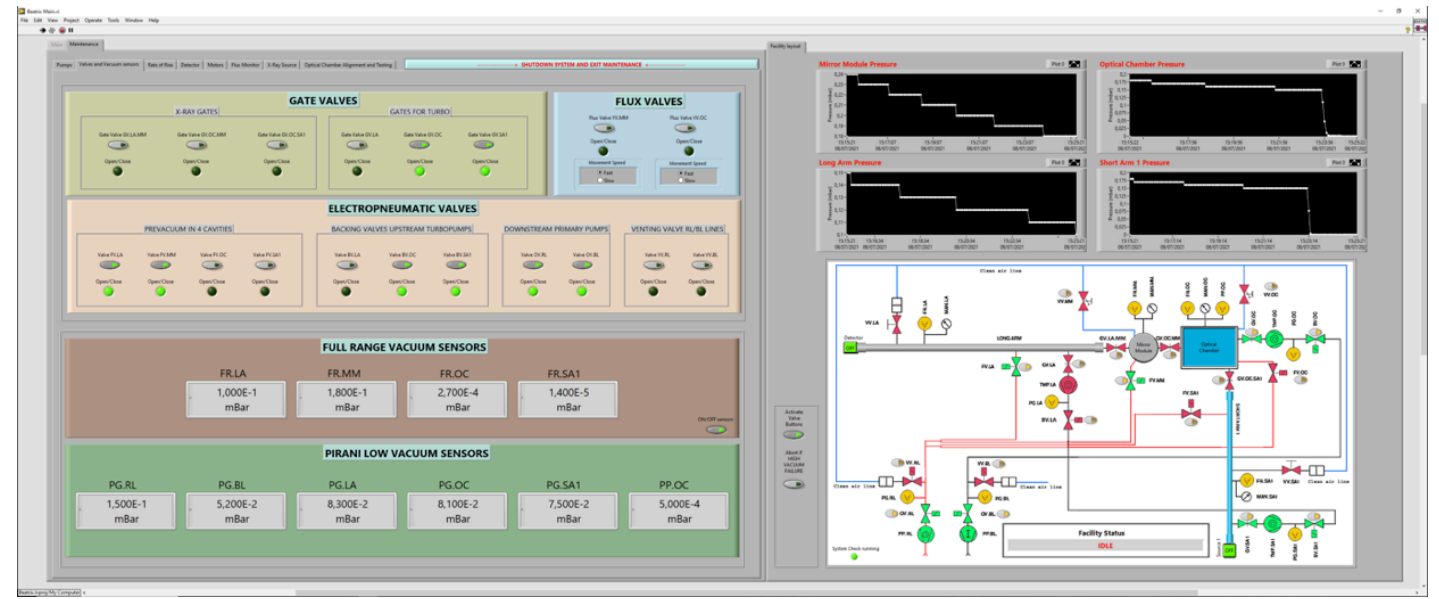

Figure 15. The "Valves and vacuum sensors" page of the LabVIEW software, and the interactive window with the facility scheme, showing in green the components under activity and in blue the sector where $10^{-4}$ mbar vacuum is reached

\section{OPTICAL COMPONENTS ALIGNMENT}

The alignment of the optical component is ongoing. At first, the X-ray source was aligned to its nominal position. To this purpose, a phosphor window was flanged at the end of the short arm (Fig. 16 A-B). The phosphor is a YAG:Ce (thickness $=0.5 \mathrm{~mm}$, diam. $=100 \mathrm{~mm}$ ) procured by CRYTUR spol. s.r.o. (Turnov, Czech Republic), which is glued to a quartz substrate (thickness $=4 \mathrm{~mm}$, diam. $=104 \mathrm{~mm}$ ). The phosphor converts X-ray photons into visible ones peaking at $550 \mathrm{~nm}$ : the conversion efficiency is $135 \mathrm{vis}-\mathrm{ph} / 4.5 \mathrm{keV}-\mathrm{ph}$. At the phosphor position, the image of the source pinhole has a diameter of $80 \mathrm{~mm}$. The BEaTriX CCD was used as a camera, flanged at the end of the Optical Chamber, and a lens (diam $=60 \mathrm{~mm}$, focal length $=130 \mathrm{~mm}$ ) was positioned in front of it to image the $80 \mathrm{~mm}$ circle of visible photons on its sensor. The alignment procedure foresees the centering of the image into the entrance aperture of the Optical Chamber (Fig. 16C), by varying the source position with the micrometers present at the source tower. 


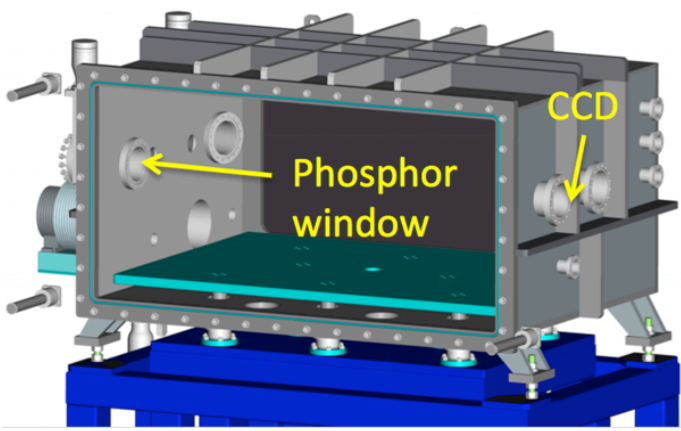

(A)

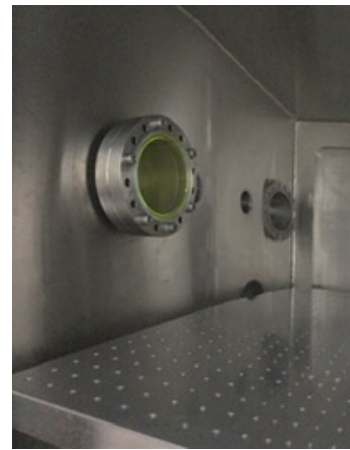

(B)

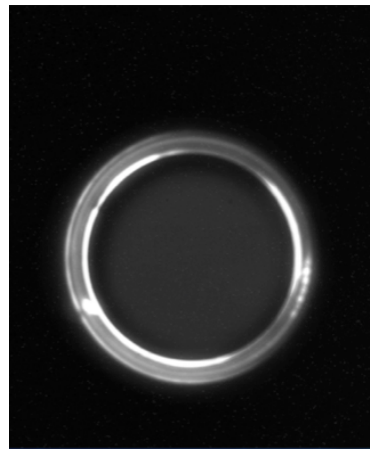

(C)

Figure 16: The phosphor window (B) is flanged at the end of the short arm, inside the Optical Chamber (A). The CCD collects the visible image $(C)$, converted from $X$-rays by the phosphors, of the aligned source.

The mirror alignment is now ongoing. At first, the mechanical alignment was performed with our 3D metrology equipment (Fig. 17 left). The mirror is placed on a reference plate, where two pinholes and two cross-hairs determine the parabola axis and the source position, respectively. The reference plate and its components are now installed in the BEaTriX Optical Chamber (Fig. 17 right). The parabolic mirror is pre-aligned with visible light, using a visible laser, and a Micro Alignment Telescope; the alignment is assisted by a Laser Tracker. Then the mirror will be aligned in Xray with the assistance of a wavefront sensor (in collaboration with LOA and Imagine Optic, France).

Once the mirror is aligned, we will start aligning the crystals. At the end, the BEaTriX X-ray beam will be qualified, placing a Hartmann plate, made in stainless steel, at the position of the MM, and measuring the displacement of the holes imaged on the CCD. If needed, a refinement of the alignment, guided by the Hartmann test results, will be performed.

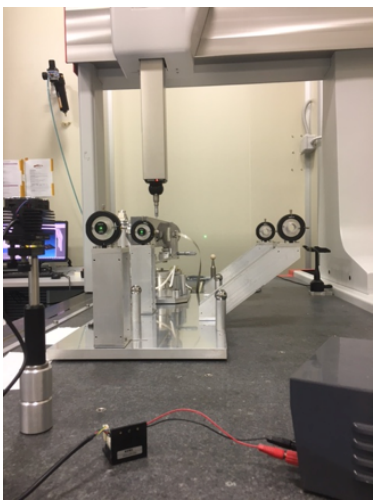

(A)

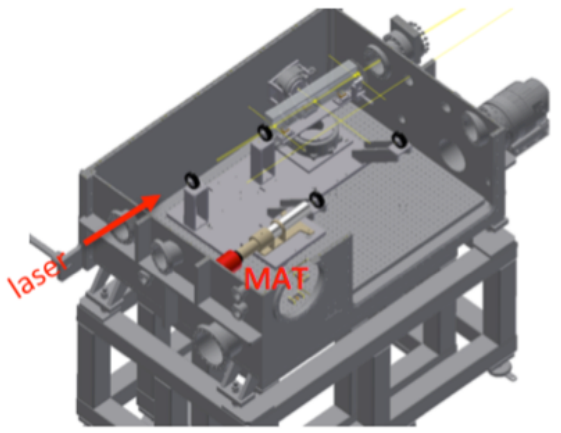

(B)

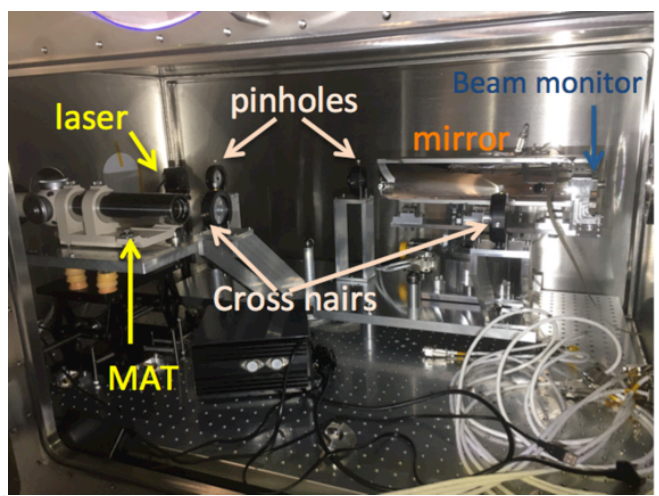

(C)

Figure 17: A) The coated mirror mounted on the reference plate, on the 3D metrology equipment. B) Sketch of the reference plate inside the Optical Chamber. The sources and mirror axes are drawn. C) The reference plate and its components mounted in the Optical Chamber.

\section{CONCLUSIONS}

The first beamline of the BEaTriX facility, operating at $4.51 \mathrm{keV}$, is almost completed at INAF-OAB, in its Merate premises. The assembly of the vacuum system started in June 2020, during the Covid-19 emergency. As everywhere else, this situation has affected the scheduling with delays and difficulties in the coordination of the works. Nevertheless, the whole system is now installed and ready for alignment. The vacuum system has full functionalities. The X-ray source was installed and aligned to its nominal position by using a phosphor window; a pinhole was installed in front of the source and measurements have shown negligible scattering from its borders; a shutter was installed in front of the source, and electrically connected to the CCD. The parabolic mirror was polished at INAF-OAB, and coated at DTU, while the X-ray calibrations were performed at PANTER before and after the coating. From the PSF, a HEW value of about 3 arcsec was computed at $1.5 \mathrm{keV}$ in parallel beam configuration. The PSF at $4.5 \mathrm{keV}$ was more 
challenging to measure and we have an upper limit estimated of 4.5 arcsec; most probably the increase is due to parts of the mirror surface that are not optically involved in the beam generation in BEaTriX. From these results, we expect a contribution of the mirror to the BEaTriX X-ray beam collimation of about $2.5 \mathrm{arcsec}$ and $1.0 \mathrm{arcsec}$ in horizontal and vertical direction, respectively. The Channel Cut Crystals in $\operatorname{Si}(220)$ were produced and qualified after a re-etching that was necessary to remove some mosaicity observed at the borders of the crystals. The asymmetric crystal, in charge of the beam expansion, was already available and qualified. The vacuum motors were installed and electrically tested with the vacuum feed-throughs. A thermal box was fabricated in INAF-OAB, installed in the MM Chamber, and tested in vacuum for reaching the necessary temperatures. The in-air motorizations for the CCD were installed and tested. The CCD was qualified in incoming and the cold finger, realized at INAF-IASF, was tested to reach the necessary temperature. A LabVEW control software was developed by $\mathrm{OAB}$, to control not only the vacuum system but also all the components of the facility. The whole facility is working under UPS, and the battery is in turn informing the computer whether a controlled shutdown is necessary. The alignment of the optical components in currently ongoing. We expect to compete the beam qualification by fall 2021 and thereafter start the tests with the ATHENA MMs.

\section{ACKNOWLEDGMENTS}

The project is financed by ESA (contract \# 4000123152/18/NL/BW), AHEAD (grant \#654215 and \#871158), ASI (grant \# 2019-27-HH.0) and INAF funds. We thank Paolo Conconi for his guidance and assistance in the project.

\section{REFERENCES}

[1] Nandra, K., Barret, D., Barcons, X., et al., "The Hot and Energetic Universe: A White Paper presenting the science theme motivating the Athena mission," http://arxiv.org/abs/1306.2307 (2013).

[2] Collon, M. J., Babic, L., Barrière, N. M., Bayerle, A., Castiglione, L., et al., "X-ray mirror development and production for the Athena telescope," Proc. SPIE 11852, 118521Z (2021).

[3] Bavdaz, M., Wille, E., Ayre, M., Ferreira, I., Shortt, B., et al., "The Athena x-ray optics development and accommodation," Proc. SPIE 11852, 1185220 (2021).

[4] Krumrey, M., Muller, P. et al., "New X-ray parallel beam facility XPBF 2.0 for the characterization of the silicon pore optics," Proc. SPIE 9905, 99055N (2016).

[5] Bradshaw, M., Burwitz, V., Hartner, G., Langmeier, A., Vacanti, G., et al., "Testing ATHENA optics: a new measurement standard at the PANTER x-ray test facility," Proc. of SPIE 11852, 1185223 (2021).

[6] Salmaso, B., et al. BEaTriX, the Beam Expander Testing X-ray facility for testing ATHENAs SPO modules: progress in the realisation, Proc. SPIE 11119, 111190N (2019)

[7] Spiga, D., Functional tests of modular elements of segmented optics for x-ray telescopes via an expanded beam facility, Proc. SPIE 8443, 84435F (2012)

[8] Vecchi, G., Cotroneo, V., Ghigo, M., Basso, S., Salmaso, B., Sironi, G., et al., "Manufacturing and testing of the X-ray collimating mirror for the BEaTriX facility", Proc. SPIE this conference.

[9] B. Menz, H. Brauninger, V. Burwitz et al., "Studying ATHENA optics with divergent and collimated x-ray beams," Proc. SPIE 9144, 91445J (2014)

[10] Sanchez del Rio, M., Cerrina, F., "Asymmetrically cut crystals for synchrotron radiation monochromators", Review of Scientific Instruments 63, 936 (1992)

[11] Christensen, F., Hornstrup, A., Frederiksen, P., et al., "Expanded beam x-ray optics calibration facility at the Daresbury Synchrotron," Proc. SPIE 2011, 540 (1994)

[12] Basso, B., et al. Thermal simulations for characterization of ATHENA Mirror Modules with a radiating box in the BEaTriX facility, Proc. SPIE 111191, 111191I (2019) 